\title{
Net circular polarization of sunspots in high spatial resolution
}

\author{
K. Ichimoto ${ }^{1}$, S. Tsuneta ${ }^{1}$, Y. Suematsu ${ }^{1}$, Y. Katsukawa ${ }^{1}$, T. Shimizu ${ }^{2}$, B. W. Lites ${ }^{3}$, M. Kubo ${ }^{3}$, T. D. Tarbell ${ }^{4}$, \\ R. A. Shine ${ }^{4}$, A. M. Title ${ }^{4}$, and S. Nagata ${ }^{5}$ \\ 1 National Astronomical Observatory of Japan, 2-21-1, Osawa, Mitaka, Toyko, 181-8588, Japan \\ e-mail: ichimoto@solar.mtk.nao.ac.jp \\ 2 Japan Aerospace Exploration Agency, Institute of Space and Astronautical Science, 3-1-1 Yoshinodai, Sagamihara, Kanagawa, \\ 229-8510, Japan \\ 3 High Altitude Observatory, National Center for Atmospheric Research, PO Box 3000 Boulder, CO80307-3000, USA \\ ${ }^{4}$ Lockheed Martin Advanced Technology Center, 3251 Hanover Street, Palo Alto, CA 94304, USA \\ 5 Kyoto University, Oiwake-cho, Kitashirakawa, Sakyo-ku, Kyoto, 606-8502, Japan
}

Received 13 November 2007 / Accepted 14 January 2008

\section{ABSTRACT}

\begin{abstract}
Context. Net circular polarization (NCP) of spectral lines in sunspots has been most successfully explained by the presense of discontinuities in the magnetic field inclination and flow velocity along the line-of-sight in the geometry of the embedded flux tube model of penumbrae $(\Delta \gamma$-effect).

Aims. The fine scale structure of NCP in a sunspot is examined with special attention paid to spatial relations of the Evershed flow to confirm the validity of the present interpretation of the NCP of sunspots.

Methods. High resolution spectro-polarimetric data of a positive-polarity sunspot obtained by the Solar Optical Telescope aboard Hinode are analysed.

Results. A positive NCP is associated with the Evershed flow channels in both limb-side and disk center-side penumbrae and with upflows in the penumbra at disk center. The negative NCP in the disk center-side penumbra is generated in inter-Evershed flow channels. Conclusions. The first result is apparently inconsistent with the current explanation of NCP with the $\Delta \gamma$-effect but rather suggests a positive correlation between the magnetic field strength and the flow velocity as the cause of the NCP. The second result serves as strong evidence for the presence of gas flows in inter-Evershed flow channels.
\end{abstract}

Key words. Sun: sunspots - Sun: magnetic fields

\section{Introduction}

Net circular polarization (NCP) defined as $\int V \mathrm{~d} \lambda$ with Stokes-V integrated over spectral lines is an important property of sunspot penumbrae that any model should account for. Observation of the NCP in sunspots was first reported by Illing et al. (1974a,b) and was followed by Henson \& Kemp (1984) and Makita \& Ohki (1986). The basic properties of NCP in sunspot are summarized as follows (e.g. Martinez Pillet 2000); 1) the largest NCP occurs in the limb-side penumbra around the apparent magnetic neutral line with the same sign as the umbra's blue lobe of the $\mathrm{V}$ profile; 2) the disk center-side penumbra also shows NCP but in the opposite sign to that of the limb-side penumbra and with less magnitude; 3 ) penumbrae of sunspots at disk center show $\mathrm{NCP}$ with the same sign as the umbra's blue lobe of the $\mathrm{V}$ profile. High resolution observation of the NCP in a penumbra is reported by Tritschler et al. (2007). They demonstrated the filamentary structure of NCP, though the spatial correlation with the Evershed flow is not conclusive.

The NCP is generated by a gradient of plasma motion along the line-of-sight (LOS) in the line forming region (Auer \& Heasley 1978). To create a reasonable amount of NCP,

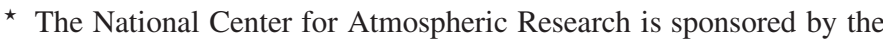
National Science Foundation. a coupling of velocity gradient with the gradient of magnetic fields is necessary. Three mechanisms are known; $\Delta B$, $\Delta \gamma$ and $\Delta \chi$ effects, i.e., coupling of plasma velocity with the magnetic field strength $(B)$, inclination $(\gamma)$ and azimuth $(\chi)$, respectively (Sanchez Almeida \& Lites 1992; Landolfi \& Landi Degl'Innocenti 1996).

The NCP of sunspots was first interpreted by the $\Delta B$-effect by Illing et al. (1975) with the increasing magnetic field strength with depth and upward mass flow in the subsurface layer of the penumbra. Makita (1986) interpreted the NCP of sunspots by the $\Delta \chi$-effect, i.e., the sunspot magnetic field is twisted and unwound along its axis, and has azimuth rotation along the LOS in penumbra. However there is no theoretical nor observational support for such velocity and magnetic field configurations of sunspots.

The most successful scenario that reproduces the NCP of sunspots today is based on the $\Delta \gamma$-effect. Sanchez Almeida \& Lites (1992) explained the NCP by a penumbral model in which the Evershed flow increases and magnetic field lines become progressively more horizontal with depth. Solanki \& Montavon (1993) proposed an "uncombed" penumbral structure to account for the gradient (or discontinuity) of the field inclination along the LOS; i.e. nearly horizontal magnetic flux tubes carrying the Evershed flow are embedded in a more inclined background 
Table 1. Used dataset and spatially averaged NCP in penumbra.

\begin{tabular}{lcccc}
\hline \hline Date & Time & $\theta(\mathrm{deg})$ & \multicolumn{2}{c}{ NCP (mA) } \\
2007 & $(\mathrm{UT})$ & & DC-side & Limb-side \\
\hline $2 / 28$ & $18: 25$ & 1.2 & 1.7 & 1.7 \\
$3 / 1$ & $23: 27$ & 11.2 & -0.4 & 5.1 \\
$3 / 2$ & $00: 42$ & 17.2 & -0.4 & 5.3 \\
$3 / 3$ & $00: 37$ & 30.4 & -0.8 & 6.9 \\
$3 / 4$ & $00: 38$ & 44.2 & -1.6 & 7.6 \\
\hline
\end{tabular}

magnetic field that wraps around the cylindrical flux tube. Those works were followed by more elaborate models by Martinez Pillet (2000) and Borrero et al. (2006). In order to explain the NCP in penumbra at disk-center, Solanki \& Montavon (1993) and Martinez Pillet (2000) assumed an upflow in the background magnetic field to make the $\Delta \gamma$-effect operate with the deeply embedded flux tubes carrying the horizontal Evershed flow. Schlichenmaier et al. (2002) and Muller et al. $(2002,2006)$ provided penumbral models that account for the two dimensional distribution of NCP in sunspots in which the $\Delta \chi$-effect also plays a role to reproduce the asymmetric azimuth distribution of NCP about the sunspot center in the FeI $15648 \AA$ line (Schlichenmaier $\&$ Collados 2002). Borrero et al. (2007) provides a magnetohydrostatic model of penumbral filaments, and reproduced the observed azimuth distribution and center-to-limb variation of NCP of FeI $6302.5 \AA$ and FeI $15648 \AA$ lines successfully.

In this paper, we present a new observation of NCP of a sunspot in visible FeI lines at $6302 \AA$ obtained with the spectropolarimeter (SP) aboard Hinode. We focus on the spatial correlation between the NCP and Evershed flow to examine the validity of the current understanding of the origin of NCP and the nature of penumbral fine structures.

\section{Observations and results}

Normal scan maps were obtained with the SP of the Solar Optical Telescope (SOT, Tsuneta et al. 2007; Suematsu et al. 2007; Tarbell et al. 2007) aboard Hinode (Kosugi et al. 2007) for a simple, positive-polarity sunspot (NOAA10944) during the period of 2007/2/28-3/4. The SP took full Stokes profiles of the pair of FeI lines at 6301.5 and $6302.5 \AA$ with a photometric accuracy of $\sim 0.1 \%$ and a spatial sampling of $0.16 \mathrm{arcsec} / \mathrm{pix}$. After applying the standard calibration procedure to each dataset, we obtained the LOS velocity from the the bisector position of the line wing (at the intensity level of $0.8 \times$ line-depth from the line core) of FeI $6301.5 \AA$, and the NCP defined by $\int V / I_{\mathrm{c}} \mathrm{d} \lambda$ (in unit of $\mathrm{mA}$ ) with the integral including both FeI 6301.5 and $6302.5 \AA$ lines.

Table 1 summarizes the analyzed dataset. The second and third columns show the time when the slit was approximately at the center of the sunspot and the heliocentric angle, respectively. The NCP spatially averaged over $\sim 6^{\prime \prime} \times 6^{\prime \prime}$ in the disk center-side and limb-side penumbrae are shown in the fourth and fifth columns. We confirm the well known polarity rule of the spatially averaged NCP and its center to limb variation (Martinez Pillet 2000).

Figure 1 shows the continuum intensity, LOS velocity and NCP of the sunspot observed on $2 / 28,3 / 2$ and $3 / 3$. The filamentary structure of the penumbra is noticeable in both LOS velocity and NCP maps. On 2007/2/28, when the sunspot was located very close to the disk center, the Dopplergram shows a number of tiny blue shifted regions in the penumbra; they are cospatial with the penumbral bright grains and are identified as the source of the Evershed flow (Ichimoto et al. 2007). Also found are strong red-shifted patches associated with a magnetic field polarity opposite to the sunspot near the outer boundary of the penumbra. The filamentary structures of NCP have a similar spatial scale as those of the intensity and LOS velocity. In the NCP maps overlaid with contours of LOS velocity (fourth column of Fig. 1), it is obvious on 2/28 that blue-shifted (upflow) and red-shifted (downflow) regions both correlate with positive NCP. The spatially averaged NCP in penumbra at disk center is mainly created in the upflow regions distributed over the penumbra.

On $2007 / 3 / 2$ and $3 / 3$, when the sunspot was located at heliocentric angles of 17.2 and $30.3 \mathrm{deg}$, the limb-side penumbra is dominated by radially elongated filamentary structures that have a positive NCP. Contrarily, both positive and negative NCP structures are equally present in the disk center-side penumbra. In the NCP maps overlaid with contours of LOS velocity, the limb-side penumbra shows that the positive NCP is associated with the Evershed flow channels (red shift). The Evershed flow channels (blue shift) in the disk center-side penumbra are again correlated with the positive NCP. Thus the spatially averaged negative NCP of the disk center-side penumbra is attributed to the inter-Evershed flow channels rather than the Evershed flow.

Figure 2 shows the variation of NCP and LOS velocity along an ellipse that traces the mid-points of the penumbra. We can confirm the correlation between NCP and LOS velocity more convincingly, i.e., the positive NCP is associated with the Evershed flow channels in both limb-side and disk center-side penumbrae and with upflows in the penumbra at disk center.

\section{Discussion}

Table 2 summarizes the observed NCP in penumbrae for both spatial average (left) and Evershed flow channels (right), and the consequences for the magnetic field gradients. The expected signs of $\mathrm{d}|B| / \mathrm{d} \tau$ and $\mathrm{d}|\cos \gamma| / \mathrm{d} \tau$ are derived from the following formulae (e.g. Solanki \& Montavon 1992);

$$
\begin{aligned}
& \operatorname{sign}(\mathrm{NCP})=-\operatorname{sign}\left(V_{\text {blue }} \frac{\mathrm{d}|B|}{\mathrm{d} \tau} \frac{\mathrm{d} v_{\text {LOS }}}{\mathrm{d} \tau}\right), \\
& \operatorname{sign}(\mathrm{NCP})=-\operatorname{sign}\left(V_{\text {blue }} \frac{\mathrm{d}|\cos \gamma|}{\mathrm{d} \tau} \frac{\mathrm{d} v_{\mathrm{LOS}}}{\mathrm{d} \tau}\right),
\end{aligned}
$$

where $\tau$ is the optical depth and $V_{\text {blue }}$ is the Stokes-V signal in the blue lobe. $v_{\text {LOS }}$ is positive for a velocity away from the observer. The inclination $\gamma$ is defined so that $\gamma=0^{\circ}$ represents a magnetic field towards the observer and $\gamma=90^{\circ}$ a transversal field. The sign of $d|\cos \gamma| / d \tau$ is converted depending on whether the magnetic field is more horizontal or not in deeply embedded flux tubes using the geometry shown in Fig. 3.

Since the sign of $\mathrm{d} \chi / \mathrm{d} \tau$ cannot be expressed in a simple way as $\mathrm{d}|B| / \mathrm{d} \tau$ or $\mathrm{d}|\cos \gamma| / \mathrm{d} \tau$ (Makita 1986), it is not included in the table. We note, however, that the degree of NCP produced by the $\Delta \chi$-effect with a reasonable change of the field azimuth along LOS is much less than observed for FeI $6302 \AA$ lines. The smallness of the $\Delta \chi$-effect in the FeI $6302.5 \AA$ line is also argued by Schlichenmaier et al. (2002). Therefore the $\Delta \chi$-effect is not likely to be the major cause of the NCP presented here.

The magnetic field gradients derived from the spatially averaged NCP in penumbrae (left three columns of Table 2) imply that the $\Delta \gamma$-effect is a natural interpretation of the sunspot's NCP since the NCP of different portions of penumbrae can be explained with a common feature, i.e., with more horizontal 


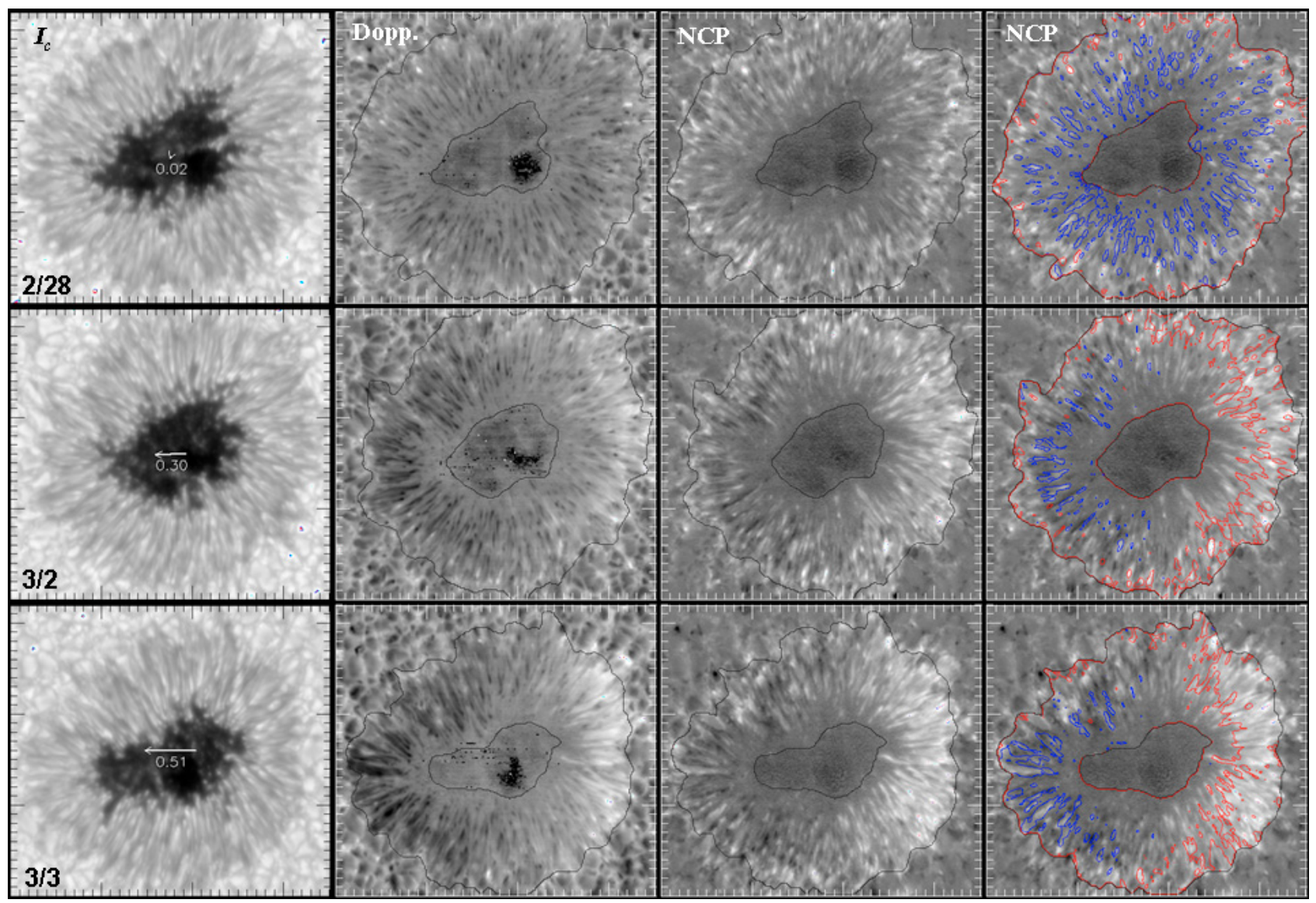

Fig. 1. Sunspot images on 2007/2/28 (top), 3/2 (middle) and 3/3 (bottom). From left to right, continuum intensity, LOS velocity, NCP and NCP overlayed with contours of LOS velocity. LOS velocity and NCP are scaled over the range of $-3 \sim+3 \mathrm{~km} \mathrm{~s}^{-1}$ and $-10 \sim+15 \mathrm{~m} \AA$, respectively. Red shift is white and the median value in the field of view is taken as the reference LOS velocity (zero line shift). Contours in fourth column are -0.8 (blue) and 0.8 (red) $\mathrm{km} \mathrm{s}^{-1}$ for $2 / 28$, and -1.6 (blue) and 0.4 (red) $\mathrm{km} \mathrm{s}^{-1}$ for $3 / 2$ and $3 / 3$. The arrow in the continuum images shows the direction to disk center with a distance in units of solar radius. Interval of minor ticks is 1 arcsec.

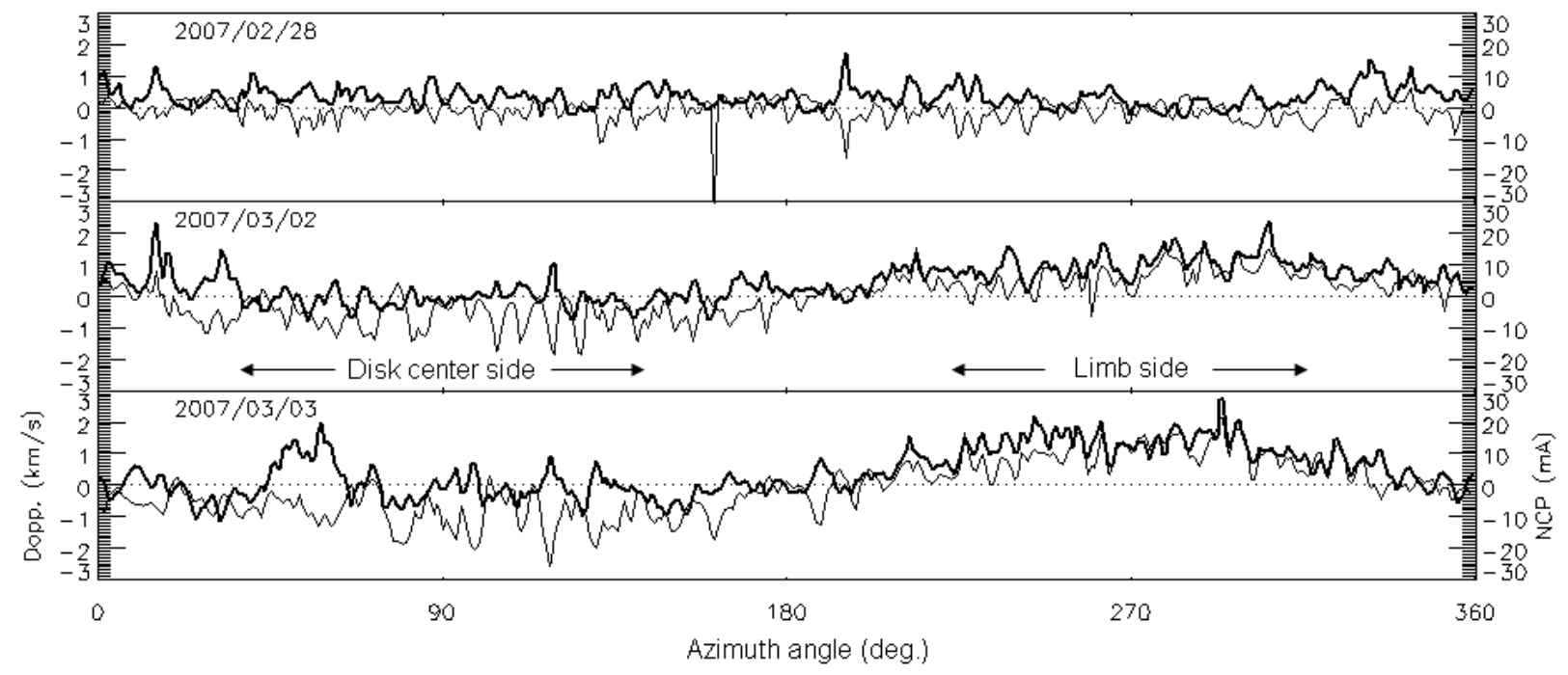

Fig. 2. NCP (thick curves) and LOS velocity (thin curves) along an ellipse tracing the approximate center of the penumbra for $2 / 28,3 / 2$ and $3 / 3$. Positive LOS velocity is red shift. The origin of azimuth angle is taken so that 90 deg. is toward the disk center and 270 deg. toward the limb.

magnetic fields of the deeply embedded flux tube carrying the Evershed flow.

According to Ichimoto et al. (2007), the positive NCP of the blue shifted regions in the disk center penumbra is attributed to a hump in the far blue wing of the Stokes-V profile which implies the presence of an upflow in the deep atmosphere. Thus $\mathrm{d} v_{\mathrm{LOS}} / \mathrm{d} \tau$ for the disk center sunspot is negative for the Evershed flow channels. The positive correlation between the 
Table 2. Observed NCP in penumbrae of positive sunspots and interpretations.

\begin{tabular}{|c|c|c|c|c|c|c|}
\hline & \multicolumn{3}{|c|}{$\begin{array}{l}\text { Average in penumbra } \\
\text { (Former picture) }\end{array}$} & \multicolumn{3}{|c|}{$\begin{array}{l}\text { Evershed flow channels } \\
\text { (This work) }\end{array}$} \\
\hline & \multirow[t]{2}{*}{ DC spot } & \multirow{2}{*}{\multicolumn{2}{|c|}{\begin{tabular}{l}
\multicolumn{2}{c}{ Off-DC spot } \\
DC-side $\quad$ Limb-side
\end{tabular}}} & \multirow[t]{2}{*}{ DC spot } & \multicolumn{2}{|c|}{ Off-DC spot } \\
\hline & & & & & DC-side & Limb-side \\
\hline \multicolumn{7}{|l|}{ Observation $^{a}$} \\
\hline NCP & + & - & + & + & + & + \\
\hline$V_{\text {blue }}$ & + & + & - & $+(-)^{d}$ & + & - \\
\hline $\mathrm{d} v_{\mathrm{LOS}} / \mathrm{d} \tau$ & $+^{b}$ & - & + & $--^{c}(+)^{d}$ & - & + \\
\hline \multicolumn{7}{|l|}{ Consequence } \\
\hline $\mathrm{d}|B| / \mathrm{d} \tau$ & - & - & + & + & + & + \\
\hline Is $B$ horizontal in deep layer? & yes & yes & yes & no & no & yes \\
\hline
\end{tabular}

${ }^{a}$ For negative polarity sunspots, signs of both NCP and $V_{\text {blue }}$ are reversed, thus the consequences are not changed. ${ }^{b}$ An upflow is assumed in the background fields (e.g. Martinez Pillet 2000). ${ }^{c}$ Upflow in deep layer (Rimmele \& Marino 2006; Bellot Rubio et al. 2006; Ichimoto et al. 2007).

${ }^{d}$ Downflow regions with opposite magnetic field polarity.

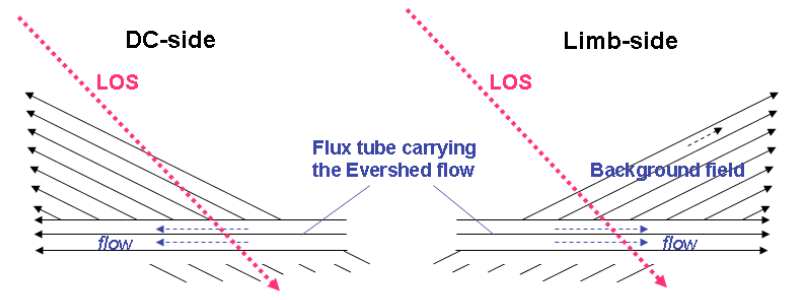

Fig. 3. Geometry of velocity and magnetic fields of the embedded flux tube model for disc center-side and limb-side penumbrae.

Evershed flow and NCP also changes the sign of the $\mathrm{d}|B| / \mathrm{d} \tau$ and $\mathrm{d}|\cos \gamma| / \mathrm{d} \tau$ for Evershed flow channels in disk center-side penumbra.

These results obviously raise the need to re-examine the current models that explain the NCP in sunspot penumbrae. For Evershed flow channels (right three columns of Table 2), the $\triangle B$-effect is apparently a more natural interpretation of the NCP of sunspots because the NCP is explained by a feature common between different portions of penumbrae, i.e., both magnetic field strength and flow velocity increase with depth. If we consider a plasma flow along a gradually expanding magnetic flux tube and assume mass continuity and lateral total pressure balance, a positive correlation between the flow velocity and field strength may be expected. However, most recent observational and theoretical studies on the filamentary structure of penumbrae predict a weaker field strength in the flux tube carrying the Evershed flow compared with the surrounding background fields (e.g. Schlichenmaier et al. 1998; Bellot Rubio et al. 2007).

A possible way to solve the contradiction may be found in the geometry of the background magnetic field that wraps around the embedded flux tube to form a cusp structure above the tube. The volume just below the cusp and above the flux tube may be a nearly field free or weak field region. When the LOS passes through this volume and the flux tube, a positive correlation between the field strength and the flow velocity is expected at the boundary of the flux tube even if the magnetic field in the flux tube is weaker than the background field in between the flux tubes. Further studies with more realistic modeling are required.

Finally, it is emphasized that the negative NCP in the disk center-side penumbra is generated in inter-Evershed flow channels. This is definite evidence for the presence of gas flows in inter-Evershed flow channels. The nature of this region is still unknown.

Acknowledgements. Hinode is a Japanese mission developed and launched by ISAS/JAXA, with NAOJ as domestic partner and NASA and STFC (UK) as international partners. It is operated by these agencies in co-operation with ESA and NSC (Norway).

\section{References}

Auer, L. H., \& Heasley, J. N. 1978, A\&A, 64, 67

Bellot Rubio, L. R., Schlichenmaier, R., \& Tritschler, A. 2006, A\&A, 453, 1117 Bellot Rubio, L. R., Tsuneta, S., Ichimoto, K., et al. 2007, ApJ, 668, L91

Borrero, J. M., Solanki, S. K., Lagg, A., Socas-Navarro, H., \& Lites, B. 2006, A\&A, 450, 383

Borrero, J. M., Bellot Rubio, L. R., \& Muller, D. A. N. 2007, ApJ, 666, L133

Henson, G. D., \& Kemp, J. C. 1984, Sol. Phys., 93, 289

Ichimoto, K., Shine, R. A., Lites, B., et al. 2007, PASJ, 59, 593

Illing, R. M. E., Landman, D. A., \& Mickey, D. L. 1974a, A\&A, 35, 327

Illing, R. M. E., Landman, D. A., \& Mickey, D. L. 1974b, A\&A, 37, 97

Illing, R. M. E., Landman, D. A., \& Mickey, D. L. 1975, A\&A, 41, 183

Kosugi, T., Matsuzaki, K., Sakao, T., et al. 2007, Sol. Phys., 243, 3

Landolfi, M., \& Landi Degl'Innocenti, E. 1996, Sol. Phys., 164, 191

Makita, M. 1986, Sol. Phys., 106, 269

Makita, M., \& Ohki, Y. 1986, Ann. Tokyo Astron. Obs., 21, 1

Martinez Pillet, V. 2000, A\&A, 361, 734

Muller, D. A. N., Schlichenmaier, R., Steiner, O., \& Stix, M. 2002, A\&A, 393, 305

Muller, D. A. N., Schlichenmaier, R., Fritz, G., \& Beck, C. 2006, A\&A, 460, 925

Rimmele, T. R., \& Marino, J. 2006, ApJ, 646, 593

Sanchez Almeida, J., \& Lites, B. W. 1992, ApJ, 398, 359

Schlichenmaier, R., \& Collados, M. 2002, A\&A, 381, 668

Schlichenmaier, R., Jahn, K., \& Schmidt, H. U. 1998, A\&A, 337, 897

Schlichenmaier, R., Muller, D. A. N., Steiner, O., \& Stix, M. 2002, A\&A, 381, L77

Solanki, S. K., \& Montavon, C. A. P. 1993, A\&A, 275, 283

Suematsu, Y., Tsuneta, S., Ichimoto, K., et al. 2007, Sol. Phys., in press

Tritschler, A., Muller, D. A. N., Schlichenmaier, R., \& Hagenaarm, H. J. 2007, submitted [arXiv:0710.4545v1]

Tsuneta, S., Ichimoto, K., Katsukawa, Y., et al. 2007, Sol. Phys., in press 